\title{
A New Fabric-based Softness Display
}

\author{
Matteo Bianchi* $\quad$ Alessandro Serio ${ }^{\dagger} \quad$ Enzo Pasquale Scilingo \\ Antonio Bicchi ${ }^{\S}$ \\ Interdepartmental Research Center "E. Piaggio", University of Pisa, via Diotisalvi, 2, Pisa, Italy
}

\begin{abstract}
This paper describes a new bi-elastic fabric-based display for rendering softness. Bi-elastic means that the fabric exhibits properties which render it elastic in at least two substantially perpendicular directions, and preferably in all directions. The device described here is based on tissue stretch to provide different levels of softness. More specifically, a thin layer of bi-elastic fabric is placed on the top of a hollow cylinder and tied to an external circular crown which can be moved up and down, relaxing or stretching the fabric. A camera placed just beneath the fabric allows for the measurement of the contact area involved in the haptic exploration. The system is also endowed with a graphical user interface, which gives a realtime visual rendering of the interaction of the fingertip with the display. In this paper, design, realization and control implementation are discussed, and performance of the display is evaluated by means of a set of psychophysical tests. We also compare performance in terms of softness perception of different simulated materials with that obtained using another softness display.
\end{abstract}

Index Terms: I.2.9 [Robotics]: Operator Interfaces-Commercial Robots and Applications.

Haptic device design and dynamics, Psychophysics and perception.

\section{INTRODUCTION}

Haptic perception allows us to explore and recognize an object by conveying several types of physical information to mechanoreceptors and thermo-receptors lying into our skin throughout the body. The term "haptic" usually refers to stimulation of both kinaesthetic and cutaneous channels $[16,12]$. In some cases kinaesthesia can play a more relevant role in discriminating physical or geometrical features rather than cutaneous information, while, in other cases, the role is symmetrically exchanged. For instance, while weight is dominated by kinaesthesia, thermal sensations are purely cutaneous.

However, both are necessary to have a fine and reliable perception of the reality, even if the cutaneous cues are generally predominant [22].

In addition to recent haptic displays [15], which focused on providing cutaneous cues, it is worthwhile mentioning other surrogating detailed contact shape information with information on the contact area on the fingertip and its changes with varying contact force (see e.g. $[8,11])$. Although all these displays are capable of rendering a reliable softness sensation, there are still some technical limitations due to low resolution of stimuli.

Here we propose a new device which conveys to subjects both cutaneous and kinaesthetic information by exploiting the bielasticity of a fabric and, at the same time, gives a direct measure-

\footnotetext{
*e-mail:matteo.bianchi@ing.unipi.it

†e-mail:xseriox@alice.it

†e-mail:e.scilingo@ing.unipi.it

§e-mail: bicchi@ing.unipi.it
}

IEEE Haptics Symposium 2010

25 - 26 March, Waltham, Massachusetts, USA

978-1-4244-6822-5/10/\$26.00 @2010 IEEE ment of the contact area involved in the interaction between the fingertip and the object.

The role of the exploration of both real and virtual textiles and fabrics is becoming an important topics in haptic research [18, 6, $24,17,23]$.

Our starting point is the hypothesis that softness discrimination by touch is given by the rate at which the contact area between manipulated objects and fingertip grows over time, as the finger is increasingly pressed on the object. This relationship, already cited and described in [8], is referred to as Contact Area Spread Rate (CASR). In [8], authors proposed a CASR display able to replicate the spread rate of contact area between probed material and fingertip. The main limitation of the CASR display presented in [8] was the low resolution of the rendered contact area. Further work [21] aimed at integrating the CASR with a commercial Delta Haptic Device [2]in order to increase performance.

The new haptic display presented here allows subjects to interact with a deformable surface at different levels of softness. In this case, haptic perception resolution is higher and discrimination capabilities are enhanced.

In this paper we discuss the design, architecture and implementation of the device. We then focus on the control mechanisms of the display based on contact area estimation. We also describe the graphical user interface, which is friendly and intuitive. The user can easily change the softness parameters to simulate as well as visualize the contact area involved during the tactile exploration.

Finally, we evaluate performance of the display through a set of psychophysical tests, and compare softness discrimination capabilities with the CASR display [8]. Results show that the subjects interacting with the new display actually seem to better discriminate softness than with the CASR display. The role of a secondary visual aid to enhance perception is also considered.

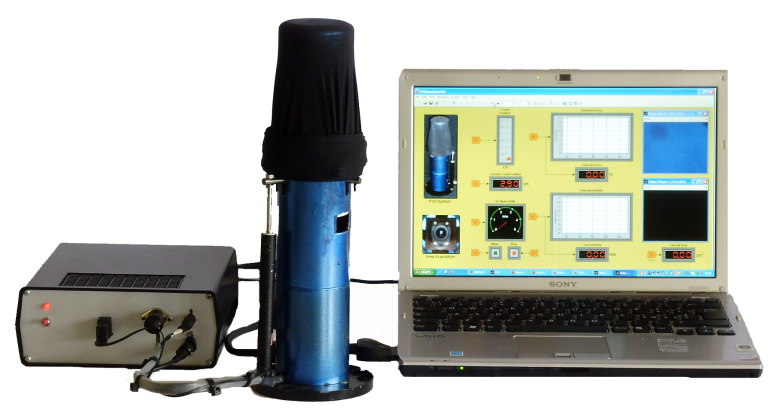

Figure 1: The system FYD: starting from the left side, it is possible to see the electronic box, which contains the motor driver and the DAQ card, the FYD prototype and a computer to control the system.

\section{FYD: STRUCTURE DESCRIPTION AND FUNCTION EXPLANATION}

The system here proposed, called hereinafter FYD (Fabric Yielding Display) is based on a layer of bi-elastic fabric which can be 
touched by subjects with their forefinger. Bi-elastic means that the fabric exhibits properties which render it elastic in at least two substantially perpendicular directions, and preferably in all directions. By changing the elasticity of the fabric, subjects are able to feel different levels of stiffness. It is comprised of a hollow plastic cylinder containing a DC motor. A thin layer of bi-elastic square shaped fabric $(250 \times 250 \mathrm{~mm})$ is placed on the top of the hollow cylinder and it is tied to a circular crown which can run outside along the cylinder, with a minimum friction. When the motor pulls down the crown, the fabric is stretched and its apparent stiffness increases. Conversely, when the motor pushes up the crown, the fabric is relaxed and it is felt softer. The FYD also behaves like a contact area display, by suitably processing signals coming from a couple of photo-devices. A view of the display is reported in fig.1.

The FYD prototype is $300 \mathrm{~mm}$ high and $60 \mathrm{~mm}$ wide in diameter and consists of three sections (see fig.2 and fig.3):

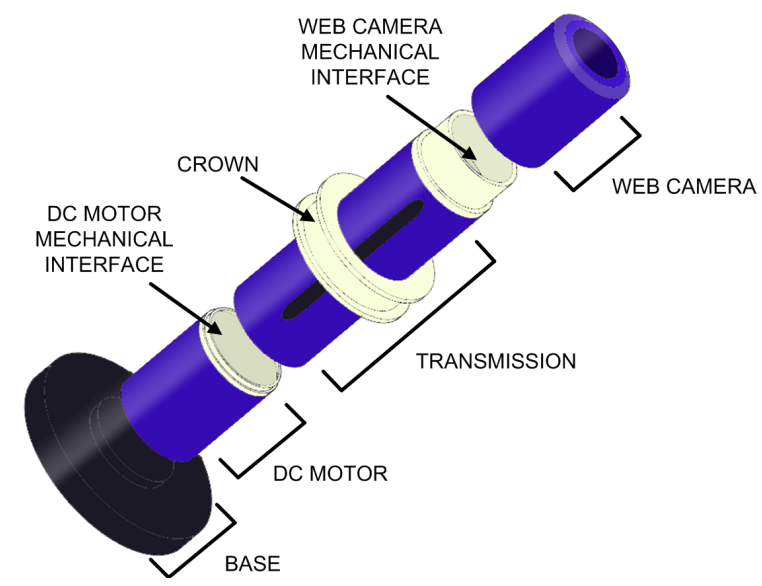

Figure 2: External view of the prototype. The potentiometric sensor connected to the crown is not reported for a better visualization.

1. Motor section ( $80 \mathrm{~mm}$ high). The motor is controlled using a Sabertooth Syren10 [1] dual motor driver. This driver allows to get a bidirectional rotation of the motor. Using the National Instrument DAQ system PCI6036E [5], we can acquire the position of the crown with an external potentiometer connected to it, and, consequently, apply the input voltage to the motor in order to reach the desired position, i.e. the desired stretching state of the fabric (see Section 2.1);

2. Transmission section (160 mm high). The transmission system converts the rotational movement of the motor into the translational movement of the crown. The system consists of a screw-female screw, the latter is attached to the crown by means of three, $120^{\circ}$ spaced, metallic supports. They are moved with the crown. The DC motor is connected to the screw with an Oldham joint, which is a homo-kinetic transmission joint, i.e. it is able to transmit the same angular position, velocity and acceleration from an input transmission shaft to an output one. The screw-female screw system is one of the principle mechanical system for the linear or translational movement. It was adopted in order to have an acceptable trade off between the velocity of the crown and the torque necessary to reach a good state of stretching of the fabric. In addition, the screw-female screw system allows a bidirectional movement of the crown;

3. Web camera section (60 mm high). The camera (whose resolution is $320 \times 240$ pixels) is placed inside the hollow cylinder at the center of the mechanical interface, just beneath the fabric. The camera is endowed with high luminosity LEDs and frames the lower surface of the fabric. During the tactual indentation, the fabric is strained and the fabric area which comes into contact with the fingertip changes according to the applied force. The camera allows to acquire the image of the strained fabric and, by means of suitable processing algorithms, the contact area can be estimated (see Section 2.2).

The prototype as a whole is connected on a base $(15 \mathrm{~mm}$ high and $90 \mathrm{~mm}$ diameter) in order to guarantee the physical stability.

Several materials (including commercial lycra, latex layer, and silicon rubber) were tested to verify their suitability for our purpose. The best performance was provided by Superbiflex HN by Mectex [4] because it exhibits both a very good elastic behavior with a large range of elasticity and a high resistance to traction.

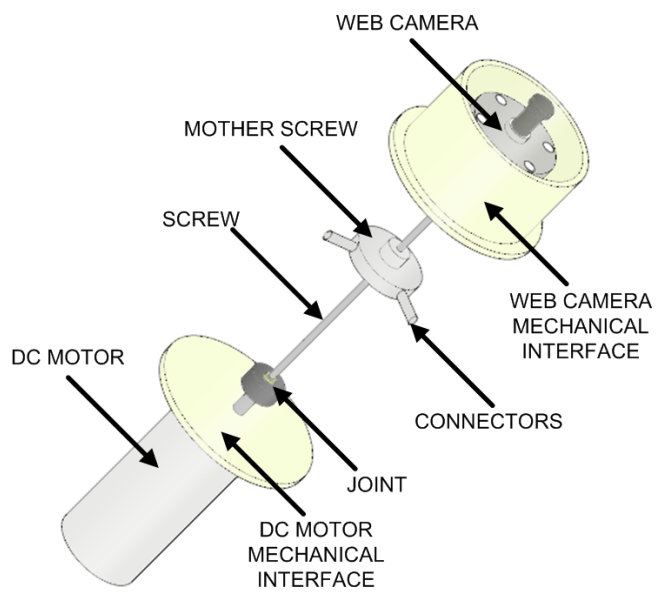

Figure 3: Prototype internal view. It is possible to see the transmission system and the DC motor position.

\subsection{Control}

Control strategy was implemented in order to have a low computational workload and guarantee a real-time functioning.

Control is based on two signals comparison (see fig.4): $P_{\text {ref }}$ and $P_{r}$ that are, respectively, the reference position and the current position of the crown read by the potentiometer.

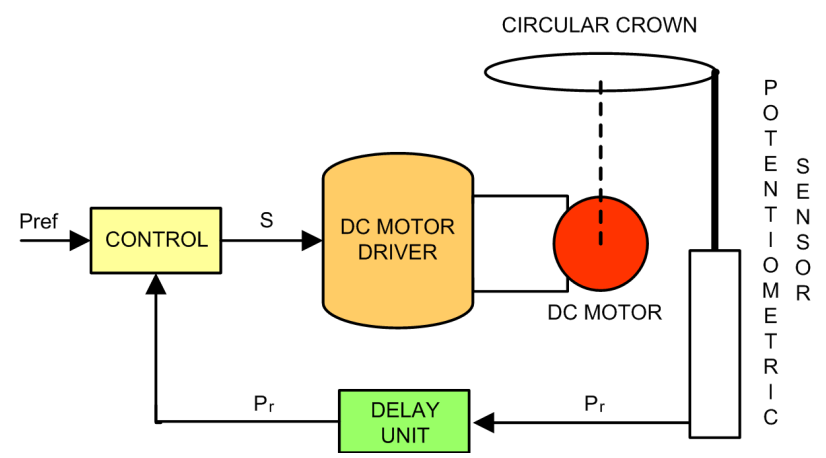

Figure 4: Control blocks architecture: $P_{r}$ is the signal acquired by the potentiometric sensor, $\mathrm{S}$ is the signal produced by the comparison of $P_{\text {ref }}$ and $P_{r}$.

The comparison produces a third signal $(\mathrm{s}$ ) for the motor driver activation. In pseudo-code the situation is represented as 


$$
\begin{aligned}
& \text { if }\left(P_{\mathrm{ref}}==P_{r}\right)\{\mathrm{s}=0\} \\
& \text { if }\left(P_{\mathrm{ref}}>P_{r}\right)\{\mathrm{s}=+1\} \\
& \text { if }\left(P_{\mathrm{ref}}<P_{r}\right)\{\mathrm{s}=-1\}
\end{aligned}
$$

The signal $\mathrm{s}$ has three logical levels. When $\mathrm{s}=-1$ the motor is driven to clockwise rotate, while when $s=+1$ the motor rotates counterclockwise. When $\mathrm{s}=0$, the DC motor is stopped at the current position. $P_{\text {ref }}$ is the position of the circular crown, to which a specific level of stretching of the fabric is associated. It is calculated from the characterization curves (see Section 2.4). $P_{r}$ is the signal recorded by the potentiometric sensor and gives the current position of the crown. At each simulation step, the control produces the signal $\mathrm{s}$ and the DC motor is moved until the actual crown position is equal to $P_{\text {ref. }}$ In fig.4 the control block diagram is reported, where the delay unit is put in to prevent algebraic loop when the control starts to run.

\subsection{Area acquisition}

The FYD system allows to visually display the contact area between the fabric and the fingertip. A suitable segmentation algorithm gives an estimation of the contact area which is visualized in real time.

The contact area acquisition algorithm is based on the RGB image binarization. More properly, only one image band (the $\mathrm{R}$ band, which is $320 \times 240$ matrix of integer numbers) out of three is involved in the area detection algorithm to avoid a computational workload too high for assuring a real-time processing.

During the tactile probing, the indented fabric surface is closer to the camera with respect to the outer region. Consequently, this area will be more lighted up by the LEDs. The difference between background luminosity and contact area luminosity is discriminated by binarization thresholds (see fig.5), which were heuristically calculated. Using a linear interpolation, at each vertical position of the crown a binarization threshold was associated. In this manner, the pixels in the image which belong to the contact area are displayed as white pixels.

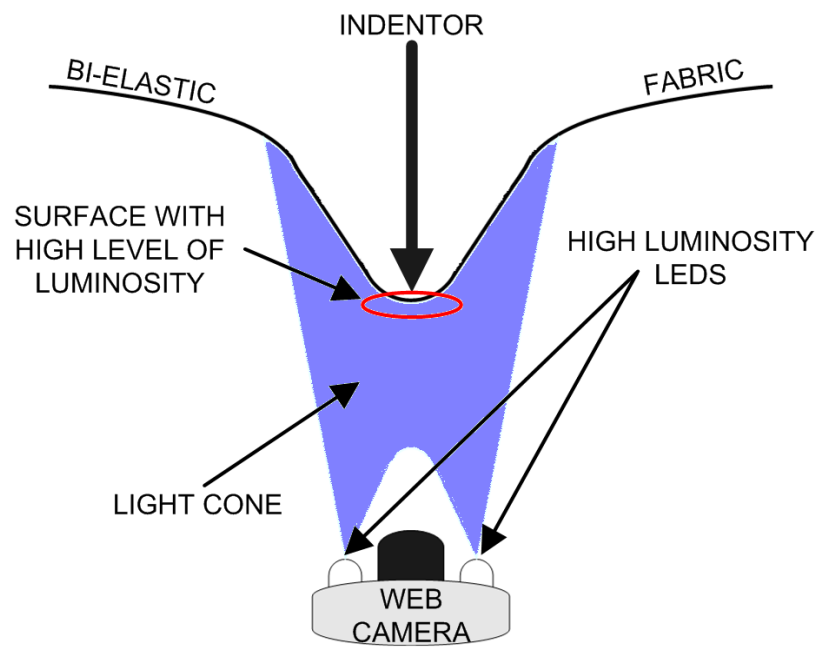

Figure 5: Scheme of the area acquisition and the illumination system.

The contact area in $\left[\mathrm{cm}^{2}\right]$ is estimated as

$$
C_{\text {area }}=N_{p} \times \frac{A_{c}}{S_{p}},
$$

where $N_{p}$ is the number of white pixels belonging to the contact area; $A_{c}$ is the frame area in $\left[\mathrm{cm}^{2}\right]$ and $S_{p}$ is the web camera res- olution (i.e. $320 \times 240$ pixels). The accuracy of the contact area measurement is crucial because the indentation force and the indentation displacement are indirectly estimated using, respectively, Force-Area and Force-Displacement characteristics. The result of the area detection algorithm is reported in fig.6.
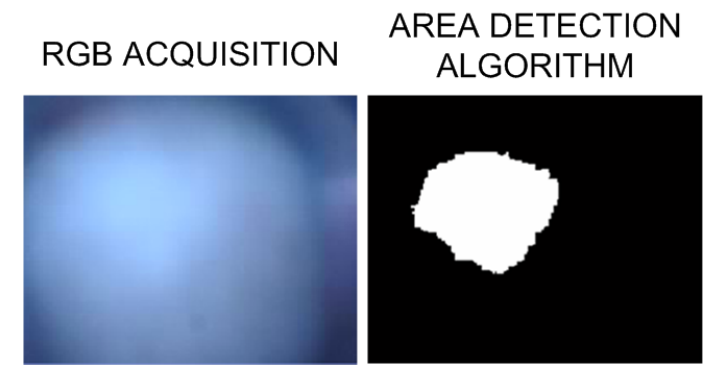

Figure 6: The results of the contact area detection algorithm: the RGB acquisition (on the left side) and the result of the final binarization (on the right side).

\subsection{Characterization and interpolation}

In this paper we disregard the contact mechanism in all its details, both from mechanical and physiological viewpoint [13], but we use a simplified model, as already proposed in [8].

As it is well known, haptic perception is given by the combination of two different modalities, kinaesthetic and cutaneous. When a haptic device is designed and realized both these perceptual cues have to be provided. In literature, there are several ways to convey these types of information. Here we use a simplified abstraction of these features.

Let the resultant contact force be denoted by $F$, the contact area by $A$. Let also $\delta$ denote the overall (rigid) relative displacement between the two bodies. The $F(\delta)$ curve of a fingertip/object pair can be considered as a close correlate of kinaesthetic information elicited by probing for softness. Analogously, starting from the CASR hypothesis [8], we will therefore consider the $F(A)$ curve of a fingertip/object pair as a correlate of cutaneous information elicited by probing for softness.

The device here proposed is controlled in order to simulate softness of materials having specific $F(\delta)$ and $F(A)$ curves. The current architecture of FYD, at least in this preliminary prototype, does not allow decoupling cutaneous and kinaesthetic information, because they are both intrinsically constrained to the elasticity of the fabric. However, by exploiting the bi-elasticity properties an acceptably large range of levels of stiffness can be simulated. The fixed coupling of $F(\delta)$ and $F(A)$ characteristics is a common limitation for the workspace of tactile devices. Therefore, each material (or device) exhibits kinaesthetic and cutaneous cues which are strongly correlated each other and, intrinsically, with the geometry and the structure of the material (or device). A possible solution to achieve an independent control of Force - Area and Force-Displacement profiles and enlarge the range of materials to be rendered could be the integration between two different haptic devices [21, 20], (see discussion about future work in Section 5).

In fig.7, the $F(\delta)$ and $F(A)$ curves of the fabric at different levels of stretching are reported. These levels were obtained changing the position of the crown, in a range between $0 \mathrm{~mm}(0 \mathrm{~mm}$ was chosen near the top of the cylinder) and $30 \mathrm{~mm}$, with an incremental step of $5 \mathrm{~mm}$. We used a load-cell to measure the force applied on the fabric during the indentation.

Indentation tests were performed by means of a compressional indentor driven by an electromagnetic actuator. The actuator is a Bruel \& Kjear minishaker, capable of applying a maximum displacement of $10 \mathrm{~mm}$ in the axial direction. The indentor is a wood 

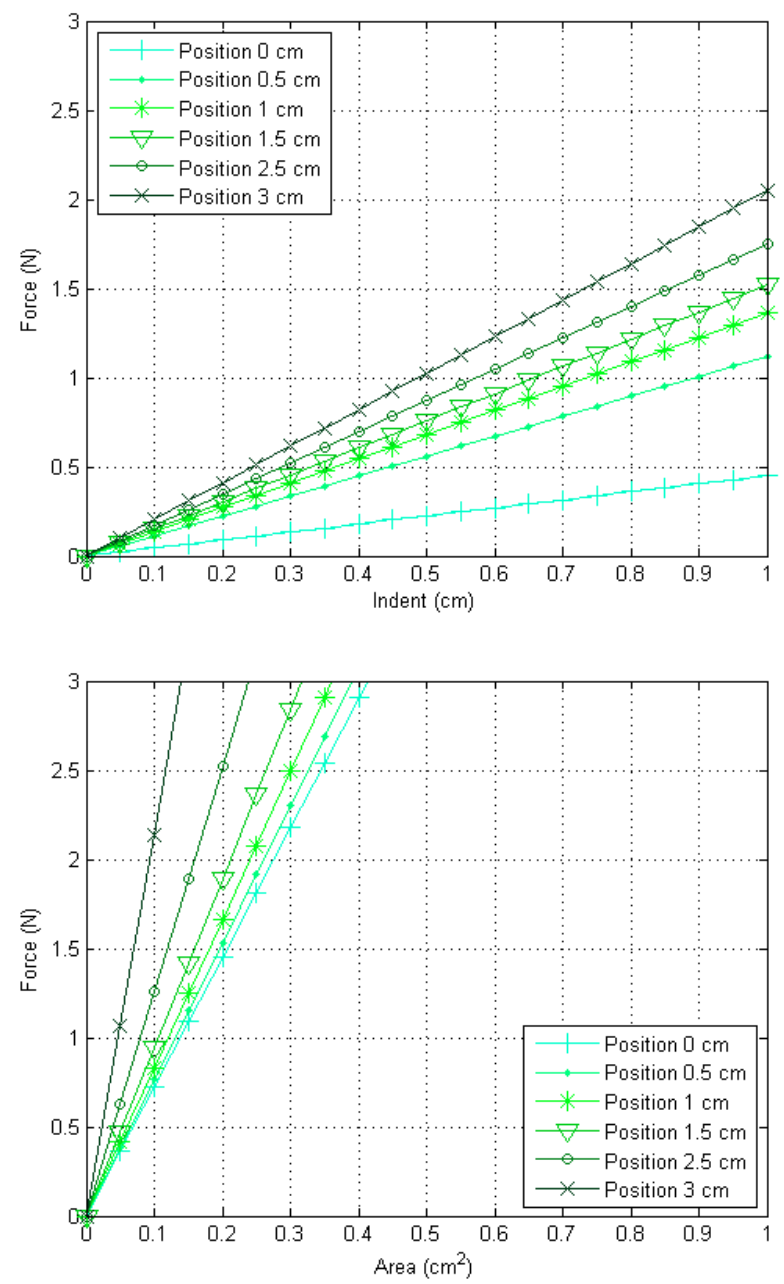

Figure 7: Characterizations of Force-Displacement (upper figure) and of Force-Area, obtained using a $5 \mathrm{~mm}$ step for the vertical crown displacement.

model of the human fingertip of $15 \mathrm{~mm}$ in diameter and $100 \mathrm{~mm}$ in length. This is a first approximation of the fingertip. Differences between the wood indentor (which is a non-compliant object) and human fingertip (which is a compliant object) should be considered. However, the deformations of the fingertip interacting with the fabric, which is naturalistically modeled under the fingertip, are very small and the approximation of the indentor with a non-deformable object is acceptable. The indentor is equipped with a magnetic linear transducer, Vit KD 2300/6C by KAMAN Science Corporation, in order to measure the vertical displacement induced on the fabric, and with a load cell sensor, ELH-TC15/100 by Entran, able to detect forces up to $\pm 50 \mathrm{~N}$. In this manner, we obtained a real-time measurement of the Force-Displacement $(F(\delta))$ characteristics for every position of the crown.

At the same time, it was possible to acquire the image of the strained fabric, by means of the camera endowed with high luminosity LEDs, placed just beneath the fabric (at a distance of $30 \mathrm{~mm}$ ). Upon suitable processing algorithms, an estimation of the contact area under the indenting force was given. In this manner, we obtained a real-time measurement of Force-Area $(F(A))$ characteristics.

During the characterization phase, only a finite set of positions was acquired. For intermediate values we used linear interpolation. When we would like to mimic a given material having a specific stiffness coefficient, we have to identify which position of the crown provides the fabric elasticity whose Force-Displacement approximates that of the material. $F(A)$ and $F(\delta)$ curves (see fig.7) are linear over all the positions of the crown.

\subsection{Graphical User Interface}

A GUI (Graphical User Interface) was implemented in MATLAB [3] to allow a correct utilization of the display. The GUI presents a hierarchical structure and consists of four windows: two for the initialization of the prototype and two for the contact area, force and indentation measurement (see fig.8).
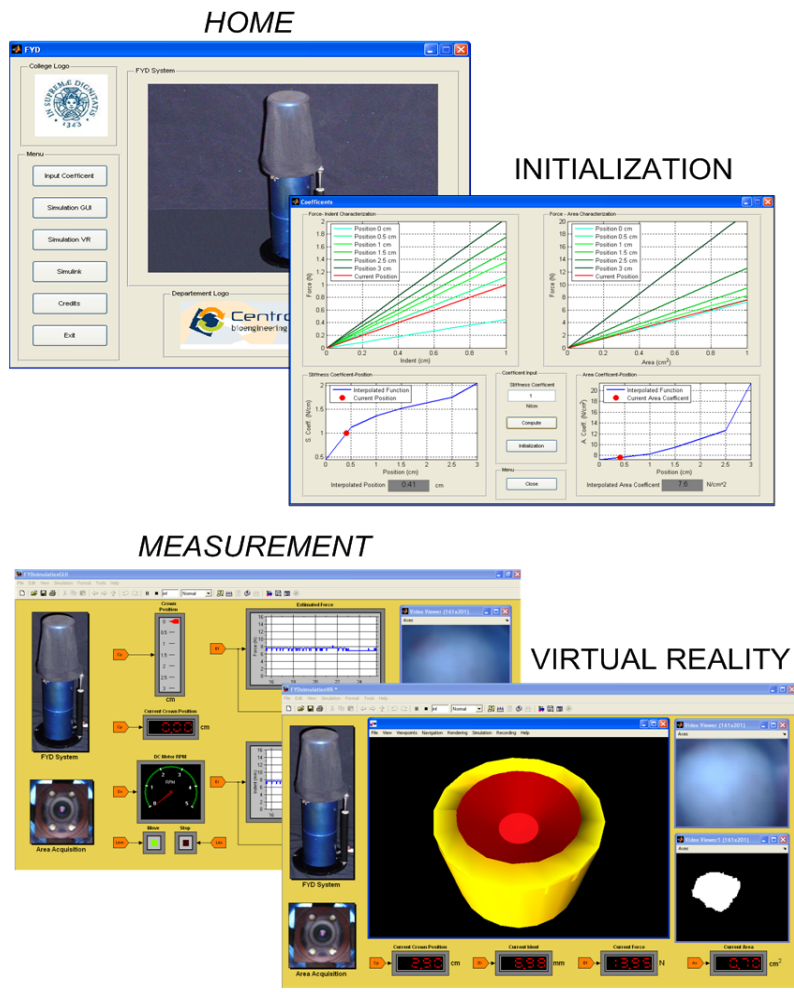

Figure 8: GUI implemented: HOME and INITIALIZATION windows for the initialization of the prototype; MEASUREMENT and VIRTUAL REALTY windows to display the measurement of force, area and indentation.

The "Home" window has the main role of managing all the simulation levels. In fact, from the "Home", each level of the GUI can be reached using an easy drop-down menu. The "Initialization" window permits to insert a stiffness coefficient for the simulation of a particular material. This stiffness coefficient corresponds to an angular coefficient of an unknown $F(\delta)$ characteristic, which can be obtained interpolating the characterization curves. At the same time, the angular coefficient describing the related $F(A)$ characteristic is calculated. Properly, in the "Initialization" window, the stiffness coefficient was compared to the Force - Indentation and Force - Area curves (which are not independent) and the corresponding vertical crown position was determined $\left(P_{\text {ref }}\right.$, see Section 2.1). The "Measurement" window offers a complete environment for the real time measurements of the principal contact parameters (e.g. contact area, force and indentation), with the possibility of visualizing both the result of the area detection algorithm and the RGB acquisition of the indented fabric. The "Measurement" allows also a control of the correct functionality of the display, in terms of 
motor performance monitoring. In the "Virtual Reality" window a simple virtual reality application was realized to geometrically describe both the contact area and the exerted indentation indirectly estimated from it (see Section 2.4.1).

\subsubsection{Virtual reality implementation}

The Virtual Reality was also implemented in MATLAB, see fig.11. To describe the geometry of the indentation, two hypotheses were assumed:

a. Contact area shape: we assumed that the contact area shape was a perfect circle with radius of $\sqrt{C_{\text {area }} / \pi}\left(C_{\text {area }}\right.$ was previously calculated using the area detection algorithm);

b. Indentation: we assumed that the axis of the indentation was the vertical axis of FYD.

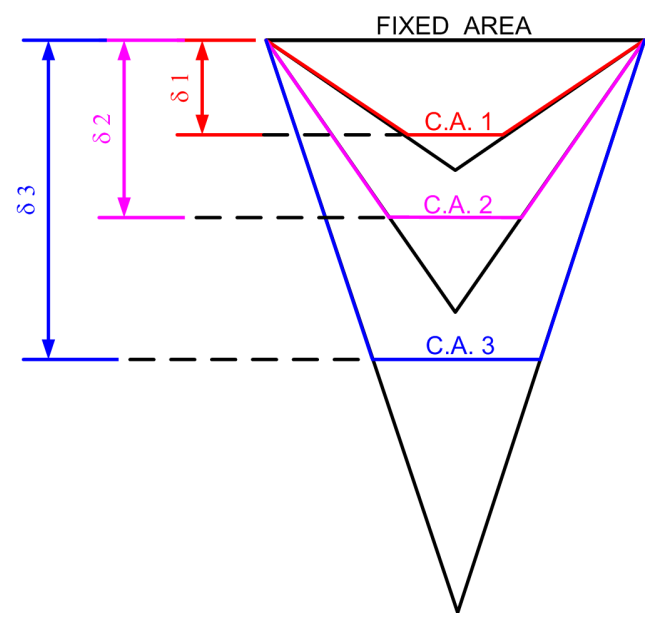

Figure 9: The set of truncated cones associated to different levels of indentation. The term "C.A." refers to the contact area. The term $\delta$ refers to the indentation.

Starting from assumptions (a) and (b), the indented surface was approximated using a set of truncated right cones nested together. They present the same area of the larger base (which is equal to the upper base of FYD), while the surface of the smaller base corresponds to the contact area and it changes according to the value of indentation. Each value of indentation was associated with a truncated cone, i.e. each value of indentation describes the height of a truncated cone, fig. 9 . In this manner, only the internal lateral surface of the truncated cone is useful for a correct visualization in a virtual environment and so visible in the virtual reality, while the rest of the cone is not displayed, even if drawn. To completely describe the lateral surface, the only parameter to determine is the total height of the cone, $h$. This parameter can be obtained by exploiting very simple geometric valuations, (see fig.10).

\section{PSYCHOPHYSICAL EXPERIMENTS}

The experimental session was designed to evaluate the performance of the FYD, comparatively with a CASR device. The performance of the FYD was assessed both with the integration of the virtual reality rendering and without it, in order to evaluate if the system improves when visual stimuli are provided. We have chosen the CASR display realized by the same authors as comparative device because it is based on the same paradigm as the FYD. Specific technical details of this CASR display can be found in [8]. Here we briefly summarize how it works. The CASR device is a pneumatic device consisting of a set of cylinders of different radius, assembled

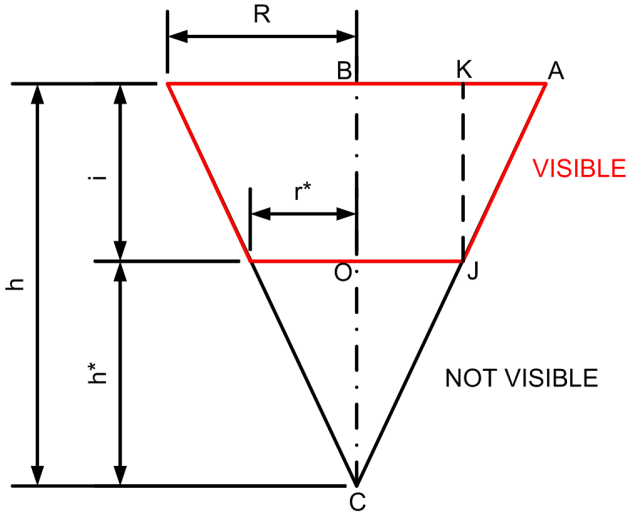

Figure 10: Cross section of a cone and some geometric parameters. $h$ is the total height of the cone (to be determined), $i$ the indentation and $R$ and $r^{*}$, respectively, the radius of the larger base and the radius of the smaller base. $h$ can be obtained by exploiting the similarity of the triangles $\widehat{K A J}$ and $\widehat{O C J}$.

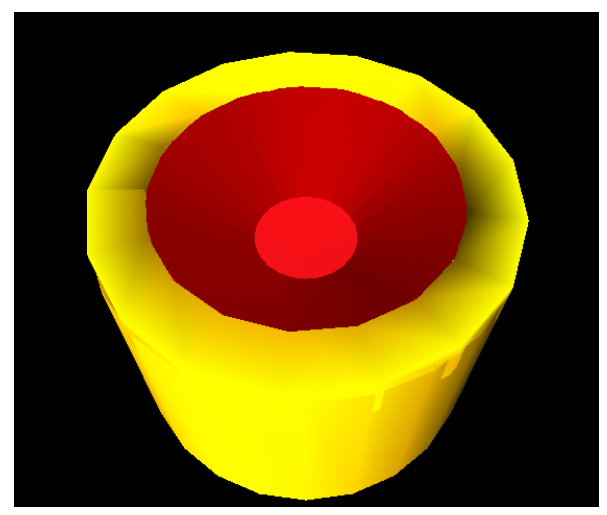

Figure 11: Final representation of the geometry of the indentation in the virtual reality.

in telescopic arrangement (see fig.12). Because of the discontinuity in the structure due to the cylinders, we will refer to this CASR display as discrete CASR display.

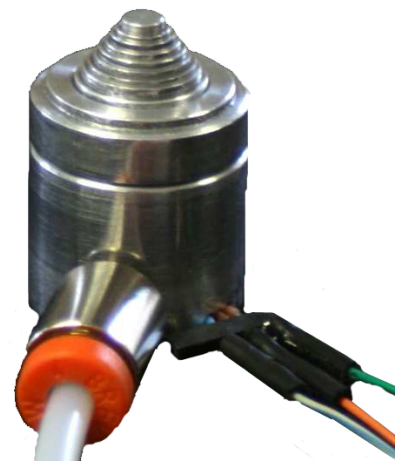

Figure 12: The discrete CASR display.

A regulated air pressure is inflated inside acting on the cylinders according to the desired force to be perceived by subjects during indentation. Pressure is applied on all the cylinders. When the subject finger pushes down against the cylinders, it comes into contact with 
a surface depending on the height of the cylinders themselves and perceives a resultant force correlated to the pressure. The display can realize a desired force-contact area (CASR) relationship [8]. Even for the discrete CASR display, the Force-Area behavior is strictly related to the geometry of the device, therefore it cannot be independently controlled from the Force - Displacement behavior.

We selected five simulated specimens, i.e. five ForceDisplacement and Force - Area characteristics, which can be exactly rendered both with FYD and the discrete CASR display. In the table 1 the input parameters of FYD and of the discrete CASR device to reproduce the five simulated specimens are reported.

Results in rendering softness with the discrete CASR display were already rather satisfactory [8]. Our aim was to improve these results using FYD.

\subsection{Subjects}

After written consensus, 10 healthy volunteers participated in the study. Their age ranged from 23 to 40 . None had a history of nerve injury or finger trauma and their finger pads were free of calluses. 5 volunteers participated only in the experiments with the discrete CASR display; 5 volunteers participated only in the experiments with the FYD display (with and without the virtual reality integration); 5 volunteers participated in all the experiments. In conclusion, each type of experiment was performed by 10 subjects. Their handedness was evaluated by the Edinburgh Handedness Inventory (EHI) [19] and they were allowed to use the dominant hand to perform the task. They always performed the tests comfortably sat, blindfolded (except for the tests performed with the integration of the virtual reality rendering, in which subjects were requested to look at a monitor visualizing the rendered tactile experience in the virtual environment) and with plugged up ears, to prevent the possible use of any other sensory cues and eliminate any diversion from the task. The chosen arm was locked to the table and the subject was able to move the wrist and fingers only.

\subsection{Rendered Specimens}

Artificial softness specimens were used through the experimental session, rendering five different Force-Displacement and Force-Area curves, see fig.7. We will refer to these specimens as $S S 1, S S 2, S S 3, S S 4$ and SS5, see tab. 1. The specimens were chosen in order to be rendered in the same way with the two displays, i.e. these specimens exhibit the same Force-Displacement and Force - Area curves both with the discrete CASR and with FYD.

\begin{tabular}{|c|c|c|c|}
\hline Stiffness & Coeff. $(\mathrm{N} / \mathrm{cm})$ & Pressure (bar) & Position $(\mathbf{c m})$ \\
\hline \hline SS1 & 0.67 & 0.35 & 0.16 \\
\hline SS2 & 1.00 & 0.5 & 0.41 \\
\hline SS3 & 1.18 & 0.6 & 0.86 \\
\hline SS4 & 1.28 & 0.7 & 1.44 \\
\hline SS5 & 1.71 & 0.8 & 2.33 \\
\hline
\end{tabular}

Table 1: Discrete CASR display (third column) and FYD (fourth column) parameters. The term "Position" refers to the vertical position of the crown of the FYD ( position 0 was chosen near the top of the cylinder) associated to a given stiffness coefficient. The term "Pressure" refers to the pressure of the air inflated into the internal camera of the discrete CASR display to mimic a given stiffness coefficient. At each value of "Position" of FYD corresponds a value of "Pressure" of discrete CASR device, in order to render the same specimens. The characteristic Force - Area is strictly related to the Force $-\delta$ curve, i.e. the stiffness coefficient.

\subsection{Experiments: Design and Procedure}

Subjects participating in these experiments were presented with rendered specimens and were asked to judge their softness by touch.
They were instructed to do so by pressing vertically or tapping the index finger of their dominant hand against the displays. Subjects were recommended not to perform movements of the finger across the surface and not to apply lateral forces. In this manner, according to the literature [14], any anisotropic effect or distortion in softness perception due to the radial/tangential discrepancy in touch is eliminated, only focusing on normal indentation of the specimens. Experiments were designed to test the ability of subjects to tactually discriminate softness both through the discrete CASR display and through the FYD, with and without the integration of the virtual reality feedback. The aim is to compare the performance in rendering specimens between these two displays. The experiments included pairwise discrimination and ranking tasks. In all tests, subjects had no time limitations and were allowed to check each specimen or haptic stimulus as many times as they wished going back and forth between them at will. The design of the experiments, in evaluating ranking and pairwise discrimination performance, is similar to the approach reported by Srinivasan and LaMotte [22], even if a direct quantitative comparison of our results with those of Srinivasan and LaMotte is not in order. Following the approach in [22], results are presented in a very clear and immediate manner, without drawing, at least at this level, any strong statistical conclusion. This methodology in data analysis refers also to the work [20], which has to appear in Transactions on Haptics. Moreover, we disregard the individuation of a threshold about JND (Just Noticeable Difference) or Weber fraction [7,9], which are fundamentally based on psychophysical considerations about human discrimination capabilities.

\subsubsection{Pairwise discrimination}

In each trial, a standard (SS3) and a comparison specimen were presented to the subjects in random order. After probing the specimens, subjects were asked to report which of the two was softer. Each task was performed three times for each subject.

\subsubsection{Ranking}

In the ranking experiment subjects were asked to probe and sort in terms of softness the set of 5 specimens $S S 1$ to $S S 5$, presented in random order. Ranking tasks were repeated three times for each subject.

\section{EXPERIMENTAL Results AND DISCUSSION 4.1 Pairwise discrimination}

Results of pairwise discrimination experiments, for both the displays, are reported in fig.13. Answers are classified as $X=1$ if the subject correctly identifies the softer specimen, or $X=0$ otherwise. The average number of correct answers $m_{n}$ is represented by the height of the histogram bars in fig.13. The number of correct answers was normalized, i.e. it was expressed in percentage terms as the ratio between the number of correct pairwise discriminations and the total number of the pairwise discriminations. Therefore, the maximum possible value assumed by $m_{n}$ was 1 , equivalent to a percentage of correct recognition of $100 \%$. The statistics of this binary experiment are described by its Bernoulli distribution. Confidence intervals for expected values $E(X)$ with statistical significance $(1-\alpha)$ are also reported in fig.13. The intervals are computed as:

$$
E(X) \in\left[m_{n}-z_{\alpha / 2} \sqrt{\frac{m_{n}\left(1-m_{n}\right)}{N}}, m_{n}+z_{\alpha / 2} \sqrt{\frac{m_{n}\left(1-m_{n}\right)}{N}}\right],
$$

with $\alpha=5 \%$, sample size $N=36$, and critical value of the normalized standard distribution $z_{\alpha / 2}=1.96$ (from standard statistical tables). It is worthwhile noting that for both the displays the normalized correct answers are very similar and comparable, for stimuli that are farther away from the reference, in the range of stiffness 
(i.e., $S S 1-S S 3$ and $S S 5-S S 3)$. For closer pairs $(S S 2-S S 3$ and especially $S S 4-S S 3$ ), artificial specimens rendered with the CASR display seem to be discriminated in a poorer way than their counterparts rendered with FYD without the virtual reality feedback. When subjects are provided with visual cues given by the contact area rendering in the virtual environment, performance appears to globally increase, though not dramatically.

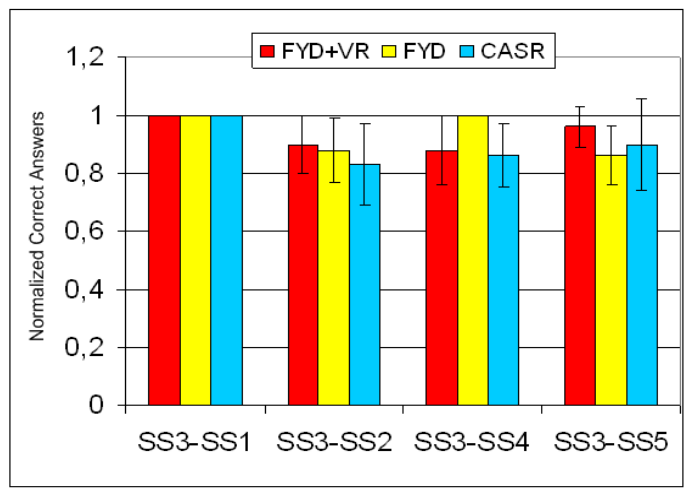

Figure 13: Results of pairwise test with CASR and FYD, with and without the virtual reality feedback.

\subsection{Ranking}

Results from ranking experiments are shown in tables 2,3 and 4, where subjective softness is reported versus objective compliance in a confusion matrix structure [22] for the five specimens, under the three different conditions. Values on the diagonal express the number of correct answers. The percentage of total accuracy is calculated considering the sum of all correct answers. The correspondence between an objective estimation of the compliance and the subjective evaluation in terms of numerical values in a given scale was already used in other work $[22,10]$. The results obtained with the discrete CASR display exhibits a percentage of total accuracy of $61 \%$. The results obtained with FYD, without virtual feedback, exhibits a percentage of total accuracy of $82 \%$. When subjects were allowed to exploit the virtual reality rendering, a total accuracy of $84 \%$ is observed. In addition, the dispersion of the matrix is reduced and the matrix appears as tri-diagonal. It means that errors are limited to pairs that are very close in the stiffness range.

\begin{tabular}{|c||c|c|c|c|c||c|c|}
\hline & SS1 & SS2 & SS3 & SS4 & SS5 & $N^{\circ}$ & Relative A. \\
\hline \hline SS1 & 18 & 4 & 1 & 1 & 6 & 30 & $60 \%$ \\
\hline SS2 & 0 & 19 & 8 & 3 & 0 & 30 & $63 \%$ \\
\hline SS3 & 2 & 1 & 18 & 8 & 1 & 30 & $60 \%$ \\
\hline SS4 & 8 & 1 & 1 & 17 & 3 & 30 & $56 \%$ \\
\hline SS5 & 2 & 5 & 2 & 1 & 20 & 30 & $66 \%$ \\
\hline \hline & & & & & & & Total A. \\
\hline$N^{\circ}$ & 30 & 30 & 30 & 30 & 30 & 150 & $61 \%$ \\
\hline
\end{tabular}

Table 2: Confusion matrix of ranking experiments with the discrete CASR display. The term "Relative A." refers to the accuracy, i.e. the percentage of correct recognition, associated to a specific specimen. The term "Total A" refers to the total percentage of correct recognition, considering all the specimens.

\subsection{Discussion}

Results show that FYD appears to provide more information than the discrete CASR display, in the pairwise discrimination tests as well as in the ranking experiments. This fact fundamentally relies

\begin{tabular}{|c||c|c|c|c|c||c|c|}
\hline & SS1 & SS2 & SS3 & SS4 & SS5 & $N^{\circ}$ & Relative A. \\
\hline \hline SS1 & 22 & 4 & 0 & 2 & 2 & 30 & $73 \%$ \\
\hline SS2 & 4 & 25 & 1 & 0 & 0 & 30 & $83 \%$ \\
\hline SS3 & 0 & 1 & 27 & 0 & 2 & 30 & $90 \%$ \\
\hline SS4 & 3 & 0 & 0 & 25 & 2 & 30 & $83 \%$ \\
\hline SS5 & 1 & 0 & 2 & 3 & 24 & 30 & $80 \%$ \\
\hline \hline & & & & & & & Total A. \\
\hline$N^{\circ}$ & 30 & 30 & 30 & 30 & 30 & 150 & $82 \%$ \\
\hline
\end{tabular}

Table 3: Confusion matrix of ranking experiments with FYD display, without the virtual reality feedback. The term "Relative A." refers to the accuracy, i.e. the percentage of correct recognition, associated to a specific specimen. The term "Total A." refers to the total percentage of correct recognition, considering all the specimens.

\begin{tabular}{|c||c|c|c|c|c||c|c|}
\hline & SS1 & SS2 & SS3 & SS4 & SS5 & $N^{\circ}$ & Relative A. \\
\hline \hline SS1 & 24 & 6 & 0 & 0 & 0 & 30 & $80 \%$ \\
\hline SS2 & 6 & 24 & 0 & 0 & 0 & 30 & $80 \%$ \\
\hline SS3 & 0 & 0 & 30 & 0 & 0 & 30 & $100 \%$ \\
\hline SS4 & 0 & 0 & 0 & 24 & 6 & 30 & $80 \%$ \\
\hline SS5 & 0 & 0 & 0 & 6 & 24 & 30 & $80 \%$ \\
\hline \hline & & & & & & & Total A. \\
\hline$N^{\circ}$ & 30 & 30 & 30 & 30 & 30 & 150 & $84 \%$ \\
\hline
\end{tabular}

Table 4: Confusion matrix of ranking experiments with FYD and the virtual reality feedback. The term "Relative A." refers to the accuracy, i.e. the percentage of correct recognition, associated to a specific specimen. The term "Total A." refers to the total percentage of correct recognition, considering all the specimens.

on the absence of edge effects during the interaction between fingertip and fabric surface. Moreover, the fabric being deformable in a controlled way under the fingertip, this new device is able to provide cues for a more reliable and realistic perception. Indeed, FYD seems to increase performance both in tasks such as ranking, which require multiple comparisons and involve haptic memory, and in tasks of pairwise discrimination between specimens which are close in stiffness, i.e. increasing "haptic" resolution.

When FYD was used with the integration of the virtual reality feedback, the best results were observed, especially in ranking experiments when "haptic" memory is integrated with "visual" memory. Visual information is related to the contact area and local deformation of the fabric, providing helpful cues in discriminating softness. This result is very encouraging and further supports the CASR paradigm.

\section{CONCLUSIONS AND FUTURE WORK}

In this paper we reported on a new bi-elastic fabric-based display for rendering softness and for a real-time contact area estimation and visualization. Subjects interact with the FYD touching a deformable surface which is naturalistically modeled under the fingertip, therefore their capability of tactually perceiving softness appears to increase.

In addition, the FYD is endowed with an optical system for an accurate measurement and a real-time estimation and visualization of the contact area. Moreover, the virtual-reality environment associated with FYD allows a good integration between haptic stimuli and visual cues, with many possible application, e.g. medical training, videogames. In this work, the performance of the display was assessed by means of psychophysical tests. The perception of softness of different FYD-simulated materials was compared with that obtained using the discrete CASR display. From our preliminary results, what can be noticed is that subjects interacting with this new haptic display actually seem to perceive different degree of soft- 
ness in a better way. Although preliminary, these results strongly encourage the authors to continue the development of this new type of tactile display. A strong statistical validation of the results is under work. Future work is aimed at miniaturizing FYD display for a possible integration with other haptic commercial device, e.g. Delta Haptic Device by Force Dimension, to achieve an independent control of Force - Area and Force-Displacement curves. Possible future developments can be finalized to introduce other sensing systems to have a real-time measurement of the displacement involved in the tactile exploration. In this manner, we can improve the control strategy.

\section{ACKNOWLEDGEMENTS}

This research is partially funded by the EU Commission under contract IST-4-027141 Immersence.

\section{REFERENCES}

[1] http://www.dimensionengineering.com.

[2] http://www.forcedimension.com.

[3] http://www.mathworks.com.

[4] http://www.mectex.com.

[5] http://www.ni.com.

[6] M. Bergamasco, F. Salsedo, M. Fontana, F. Tarri, C. A. Avizzano, A. Frisoli, E. Ruffaldi, and S. Marcheschi. High performance haptic device for force rendering in textile exploration. The Visual Computer, Springer Berlin/Heidelberg, 23(4):247-256, April 2007.

[7] W. M. Bergmann Tiest and A. M. L. Kappers. Cues for haptic perception of compliance. IEEE Transactions on Haptics, 2(4):189-199, October-December 2009.

[8] A. Bicchi, D. E. De Rossi, and E. P. Scilingo. The role of the contact area spread rate in haptic discrimination of softness. IEEE trans. on Robotics and Automation, 16(5):496-504, October 2000.

[9] F. K. B. Freyberger and B. Färber. Compliance discrimination of deformable objects by squeezing with one and two fingers. In EuroHaptics 2006, pages 271-276, Paris, France, July 2006.

[10] R. M. Friedman, K. D. Hetster, B. G. Green, and R. H. LaMotte. Magnitude estimation of softness. Exp Brain Res., 191(2):133-142, November 2008.

[11] K. Fujita and H. Ohmori. A new softness display interface by dynamic fingertip contact area control. In 5th World Multiconference on Systemics, Cybernetics and Informatics, pages 78-82, Orlando, Florida (USA), July 2001.

[12] R. S. Johansson and G. Westling. Roles of glabrous skin receptors and sensorimotor memory in automatic control of precision grip when lifting rougher or more slippery objects. Experimental Brain Research, 56(3):550-564, October 1984.

[13] K. O. Johnson. The roles and functions of cutaneous mechanoreceptors. Curr. Opin. Neurobiol., 11(4):455-461, August 2001.

[14] S. L. Lederman and R. L. Klatzky. Relative availability of surface and object properties during early haptic processing. Journal of Experimental Psychology: Human Perception and Performance, 23(6):1680-1707, December 1997.

[15] V. Levesque, J. Pasquero, V. Hayward, and M. Legault. Display of virtual braille dots by lateral skin deformation: Feasibility study. ACM Trans. on Applied Perception, 2(2):132-149, April 2005.

[16] J. M. Loomis and S. J. Lederman. Tactual perception. Hand-book of Perception and Human Performance, II, p. 1-41. Wiley-Interscience, Malden MA, USA, 1986.

[17] C. Luible, M. Varheenmaam, N. Magnenat-Thalmann, and H. Meinander. Subjective fabric evaluation. In Int. Conference on Cyberworlds, HAPTEX'07 Workshop, IEEE Computer Society, pages 285-291, Hannover, Germany, October 2007.

[18] N. Magnenat-Thalmann, P. Volino, U. Bonanni, I. R. Summers, M. Bergamasco, F. Salsedo, and F. E. Wolter. From physics-based simulation to the touching of textiles: The haptex project. The International Journal of Virtual Reality, 6(3):35-44, September 2007.

[19] R. C. Oldfield. The assessment and analysis of handedness: The edinburgh inventory. Neuropsychologia, 9(1):97-113, March 1971.
[20] E. P. Scilingo, M. Bianchi, G. Grioli, and A. Bicchi. Rendering softness: Integration of kinaesthetic and cutaneous information in a haptic device. Transaction on Haptics, to appear.

[21] E. P. Scilingo, N. Sgambelluri, G. Tonietti, and A. Bicchi. Integrating two haptic devices for performance enhancement. In World Haptics Conference, pages 139-144, Tsukuba, Japan, March 2007.

[22] M. A. Srinivasan and R. H. LaMotte. Tactile discrimination of softness. Journal of Neurophysiology, 73(1):88-101, June 1995.

[23] M. Varheenmaam and H. Meinander. Mechanical properties as a base for haptic sensing of virtual fabrics. In Proc. Autex 2007 Conference, Tampere, Finland, June 2007.

[24] P. Volino, P. Davy, U. Bonanni, C. Luible, N. Magnenat-Thalmann, M. Mkinen, and H. Meinander. From measured physical parameters to the haptic feeling of fabric. The Visual Computer, Springer Berlin/Heidelberg, 23(2):133-142, February 2007. 